\author{
Stephanie Montrose*, MS, DO, Mark Vogel, PhD and Kimberly R. Barber, PhD
}

\title{
Use of osteopathic manipulative treatment for low back pain patients with and without pain medication history
}

https://doi.org/10.1515/jom-2019-0193

Received July 15, 2019; accepted June 16, 2020;

published online January 29, 2021

\section{Abstract}

Context: Low back pain is one of the most frequent diagnoses in primary care, and prescription pain medication is commonly used for management. Osteopathic physicians may use osteopathic manipulative treatment (OMT) as an additional tool to help alleviate pain.

Objective: To determine if nonpharmacological options can improve back pain with the use of OMT.

Methods: Two groups were studied: patients receiving OMT but not using prescribed pain medications (OMT-only group) and patients who received prescribed pain medication and began receiving OMT after three months of pharmacologic therapy (OMT + medication group). All patients were enrolled in the study for one year. The amount of time between treatments was determined by the physician performing the OMT and the patient's pain improvement. The Keele STarT survey and Oswestry Disability Index tool were used at each appointment to assess the patient's functionality and pain.

Results: Thirty-six patients enrolled in the study: 26 in the OMT-only group and 10 in the OMT + medication group. Each group reported improvement in low back pain (LBP) according to both scales used. The OMT-only group reported improvement according to the Keele STarT survey (30\% relative decrease in the mean score) and the Oswestry Disability Index tool (18\% relative decrease in disability index), while patients in the OMT + medication group also

*Corresponding author: Stephanie Montrose, MS, DO, Department of Family Medicine, Ascension Genesys Hospital, 1 Genesys Pkwy, Grand Blanc, MI 48439, USA, E-mail: smsimon10@gmail.com

Mark Vogel, PhD, Department of Family Medicine, Ascension Genesys Hospital, Grand Blanc, MI, USA

Kimberly R. Barber, PhD, Department of Research, Ascension Genesys Hospital, Grand Blanc, MI, USA reported improvement according to the Keele STarT survey (29.5\% relative decrease in the mean score) and the Oswestry Disability Index tool (18\% relative decrease in disability index). A decrease in Cyclobenzaprine usage was also observed in the OMT + medication group.

Conclusion: Both groups showed significant decreases in overall pain, and this similar effect in each group may indicate a lack of need for medications when OMT is used. Additional research on efficacy of OMT in this patient population is needed with larger, multicenter, randomized trials.

Keywords: back pain; functionality; OMT; opioid epidemic; opioids; osteopathic manipulative treatment; pain.

Chronic low back pain (LBP) is defined as pain located below the costal margin and above the inferior gluteal folds lasting greater than three months. ${ }^{1}$ It commonly leads to a decrease in function in an individual, decreased participation in a social life, and is one of the leading diagnoses in primary care. ${ }^{2}$ Current therapies for back pain include, but are not limited to, osteopathic manipulative treatment (OMT), pharmacotherapy, physical therapy, and ultrasonography. ${ }^{3}$

With the use of pharmacotherapy for pain control, an even larger problem has surfaced-an opioid epidemic. ${ }^{4}$ More than 130 people each day die of an opioid overdose. ${ }^{4}$ The Centers for Disease Control and Prevention estimates that the total "economic burden" of prescription opioid misuse alone in the United States is $\$ 78.5$ billion a year, including the health care costs, lost productivity, addiction management, and criminal justice involvement. ${ }^{4}$ The question becomes, how can primary care physicians help? What options are available (to be used alone or in complementary fashion) to use pain medications as the sole remedy for chronic LBP?

One option is OMT, a system of hands-on techniques used to help alleviate pain and improve function. OMT is defined as the therapeutic application of manually guided forces by an osteopathic physician to improve physiologic function or support homeostasis that has been altered by 
somatic dysfunction. ${ }^{5}$ Current American Osteopathic Association guidelines recommend using OMT for patients with LBP. ${ }^{3}$ Previous literature reviews examining studies of OMT for patients experiencing LBP who underwent surgery $^{6}$ and those who did not undergo surgery ${ }^{7,8}$ have been equivocal as to the efficacy of OMT. One study demonstrated a positive effect in adult surgical patients and others showed no difference between OMT and controls. ${ }^{6-8}$ A review using the Cochrane Database, published in 2012, showed that OMT was no more effective for acute LBP than inert interventions, sham OMT, or other interventions when added to other recommended therapies. ${ }^{8}$ However, several studies in that review had small samples, reported follow-up times that widely varied (one week-12 months), and were of low quality evidence. The finding from that review did highlight that among the studies using OMT as an add-on therapy, there was a moderate, clinically relevant improvement regarding on functional status when OMT was added to another intervention.

The most comprehensive review of OMT for LBP was published by Franke et al. ${ }^{7}$ in 2014 . Inclusion criteria in that systematic review consisted of randomized clinical trials of adults with nonspecific back pain treated by osteopathic physicians who used their clinical judgment as opposed to a standard predetermined protocol. Studies with pregnant and postpartum participants were also included, outcomes reported included improvement in pain and function, and the studies were evaluated for level of evidence. Among those with moderate or higher level of validity, there was evidence of significant improvement by OMT in pain and in function in acute and chronic LBP.

Previous experimental studies have examined OMT for chronic pain in both a control group of patients ${ }^{9-11}$ and sham OMT study patients. ${ }^{12,13}$ Heinze et al. showed that OMT was a suitable therapy for subacute LBP. That study looked at OMT + physiotherapy and physiotherapy alone, finding a 2.5 -fold improvement in the OMT + physiotherapy group compared with the control group. ${ }^{14}$ In a double-blind, randomized, sham-controlled trial to evaluate myofascial release in patients with back pain for longer than three months, Arguisuelas et al. ${ }^{11}$ demonstrated small but clinically significant improvements for both short- and long-term regimens in pain level, disability, and fear avoidance beliefs. These patients were all allowed to continue their pain medication regimen and no other information about their medication was retrieved. In a study examining OMT as a complement to usual care (advice, muscle relaxants, and passive modalities such as ice), Cruser et al. ${ }^{10}$ compared OMT + usual care with usual care alone for patients with acute LBP in active duty military personnel. Patients were randomly assigned to groups and received four treatment sessions. After a four-week post-trial period, patients with OMT + usual care showed greater improvement. ${ }^{10}$

In a study designed to address the limitations inherent in previous OMT clinical trials, Licciardone et al. completed a randomized, double-blind, double shamcontrolled $2 \times 2$ factorial study of OMT for chronic LBP. ${ }^{15}$ With 455 enrolled participants, it is possibly the largest OMT trial for patients with LBP published to date. Six OMT sessions were provided to patients over an eight week period with outcomes at 12 weeks. The primary outcome was a substantial (greater than 50\% reduction) improvement in pain, with functional status as a secondary outcome. No significant improvements were seen among patients who began the study with lower pain severity. However, among those with high pain severity at baseline, there were significant improvements demonstrated for the OMT group. The OMT patients achieved substantial improvements in pain and significant benefits in all secondary measures including function. Of note is that patients in the high baseline severity group were more likely to be on prescription pain medications when they started the study. Even so, $64 \%$ of the OMT patients vs. $29 \%$ of the sham OMT patients achieved substantial improvement in LBP (relative risk, 2.2; 95\% confidence interval, 1.5-3.3; $\mathrm{p}<0.001)$. That study demonstrated large effect-size improvements by OMT in providing substantial pain reduction for chronic LBP patients with high severity pain, many of whom were on pain medications. Thus, the results supported OMT as a rational, evidence-based option for patients with severe LBP before more invasive management options.

While a review by Franke et al. ${ }^{7}$ included studies that compared OMT-only groups to OMT + pain medication groups, none were longer than 90 days. In assessing the impact of OMT for LBP, it is important to examine patients already receiving pharmacotherapy and those not using any pharmacotherapy because LBP is so prevalent in the ambulatory world, and pharmacotherapy is commonly used to manage LBP.,16 The concern with pharmacotherapy as first-line treatment is that pain is masked, so the true cause may not be addressed or corrected. If somatic dysfunction, which is amenable to OMT, is the cause (or a component of the cause) of the LBP, then it should be assessed as part of the differential diagnosis and treated with or without the use of pharmacotherapy.

Therefore, we conducted a pragmatic, communitybased pilot study at an osteopathic family medicine residency clinic to determine whether chronic LBP can be improved with OMT alone or as a complement to pain 
medications. We hypothesized that the use of OMT in patients not using prescribed pain medication would have similar improvements in pain and functionality compared to those receiving OMT and pain medication.

\section{Methods}

\section{Study design}

This Ascension Genesys Institutional Review Boardapproved pilot study was a prospective, nonrandomized, nonblinded investigation completed at an ambulatory residency clinic. The study period occurred between October 1, 2017 and February 1, 2019. Written consent for the study was obtained from all participating patients. Two different groups of patients were examined in the study, all of whom had chronic back pain: those who were already using pharmacotherapy for their back pain (OMT + medical group) and those who were not using any prescribed pharmacotherapy for their back pain (OMT-only group). Patients in the OMT-only group had not received OMT in the three months prior to enrollment (or never at all) and were not using prescribed pharmacotherapy for back pain. Patients in OMT + medication group had already received prescribed medication for their back pain but had not received OMT in the three months prior to enrollment (or never at all).

Patients in the OMT-only group started receiving OMT upon enrollment. These patients saw their physician at intervals determined by the physician and patient. These intervals were based on improvement of pain and function between treatments. OMT was provided for a period of one year. Opioid use was recorded for both groups. The protocol (as stated on the consent form) indicated that if patients in the OMT-only group needed to be prescribed pain medication during the duration of the study, they were to be removed from the study, but no patients met these criteria during the study period. OMT + medication group patients were titrated on their medication for three months until a therapeutic level was determined by the physician and patient together according to the improvement in pain felt by the patient. At three months, these patients started receiving OMT. Treatments were based upon improvement in pain and function. The OMT + medication group continued OMT for nine months. If a patient reported pain improvement during this nine-month period, the physician would titrate down pharmacotherapy. The type of treatment performed was based on the dysfunction that the patient experienced. Different techniques of OMT were used throughout the study depending on the specific somatic dysfunction found in the patient. ${ }^{1,17}$

\section{Subjects}

Selection of patients was based on a mixed sampling method. Adult patients in an ambulatory family medicine residency clinic were approached for participation. About 300 patients were initially identified through a medical records search using ICD-10 codes for back pain. All of these patients were then mailed a flyer about the study inquiring whether they wanted to participate in a back pain study and to contact their physician to determine whether they qualified for inclusion. This same flyer was then placed in multiple locations within the ambulatory family medicine site to recruit more participants. If a patient came in for back pain or if a diagnosis of back pain was known to the resident physician seeing the patient, the resident physician could tell the patient about the study and ask if they would like to participate, pending the patient meeting inclusion criteria.

To be included, patients needed to be between the ages of 18 and 75 years and have had LBP for at least six months. Diagnosis of somatic dysfunction was based on physician palpation of the patient's back and included changes in tissue texture, increased sensitivity to touch, altered ease or range of movement, and anatomic asymmetry or positional change per American Association of Colleges of Osteopathic Medicine. ${ }^{5}$

Exclusion criteria were use of OMT in the previous three months, chiropractic care in the previous three months, steroid use in the previous three months, current metastatic cancer, or pregnancy. Patients signed a consent form before joining the study for a one-year time period if they met the inclusion criteria. Personal identification information was protected following HIPAA guidelines by using subject study numbers and a separate master list of patient names.

\section{Procedures}

OMT providers included family medicine osteopathic resident physicians who were overseen by attending physicians at each appointment. There were 10 different physicians performing the OMT on patients. Some patients saw the same physician each time, while others saw a different physician. The techniques used were counterstrain, muscle energy, myofascial release, and high velocity low amplitude. 
Table 1: Clinical assessment outcomes over time by group.

\begin{tabular}{lrrrr}
\hline Outcome & \multicolumn{2}{c}{ Before treatment, mean score (SD) } & \multicolumn{2}{c}{ End of treatment, mean Score (SD) } \\
\cline { 2 - 5 } & \multicolumn{1}{c}{ OMT-only } & OMT + medication & OMT-only & OMT + medication \\
\cline { 2 - 5 } Keele STarT & $6.3(2.4)^{\mathrm{a}}$ & $7.8(2.4)^{\mathrm{a}}$ & $4.5(2.3)^{\mathrm{c}}$ & $5.5(2.6)^{\mathrm{c}}$ \\
Oswestry & $46.1(14.9)^{\mathrm{b}}$ & $62.9(15.5)^{\mathrm{b}}$ & $37.8(11.6)^{\mathrm{d}}$ & $51.3(18.0)^{\mathrm{d}}$ \\
\hline
\end{tabular}

Between group comparisons: ${ }^{a} p=0.103 .{ }^{b} p=0.005 .{ }^{c} p=0.59 .{ }^{d} p=0.43$. Keele STarT, Keele Subgroups for Targeted Treatment back screening tool; OMT, osteopathic manipulative treatment; Oswestry, Oswestry Disability Index; SD, standard deviation.

\section{Pain and function assessments}

Two surveys, the Keele STarT ${ }^{18}$ and the Oswestry Disability Index, ${ }^{19}$ were used to assess pain and function of the patients and were given at each appointment. A form asking for pain medication type, dosage, and frequency was also completed. The resident physician informed patients about the surveys during their first appointment to ensure they were understood. After the first appointment, resident physicians would give the patient the surveys to be completed at the end of the appointment after the physician left the room. The patients left the surveys in the room when finished, and medical assistants would then pick up surveys and place them in a designated secured folder after the patient had departed. This method was designed to make sure patients did not feel pressure when completing the surveys.

Keele STarT back screening tool is a brief, validated tool, designed to screen patients with LBP for prognostic indicators that are relevant to decision making. ${ }^{17}$ This survey was used to assess pain in our patients. A modified Oswestry Disability Index was also used, with six questions instead of 10, to assess the functionality of the patient. The sections used were sleeping, standing, sitting, sex life, traveling, and social life. This was done by taking the sum of the score in each section and multiplying by 3.33 to obtain a scaled score then ranged from 0 to 100 . The scale was further categorized into disability sections: minimal (0-20\%), moderate (21-40\%), severe (41-60\%), crippled (61-80\%), and bedbound (81-100\%). A third survey was given that asked patients about their usage of pain medication, in which they were asked to report type, dosage, and frequency.

Descriptive parametric data, as well as muscle relaxer use and medication dosage, were analyzed using means and standard deviations (SD). Descriptive nonparametric data were analyzed using medians (range) and frequency counts (percentages). Number of visits was compared between groups with a Mann Whitney $\mathrm{U}$ t-test for independent groups. Back disability outcomes based on categories of risk (Keele STarT) were compared between groups with $\chi^{2}$ analysis. Back disability outcomes based on rank sums (Oswestry Disability Index) were compared using the Mann Whitney U t-test. Frequency of cyclobenzaprine prescription was recorded to determine whether there was any improvement. Oswestry Disability Index and Keele STarT means were calculated at the start

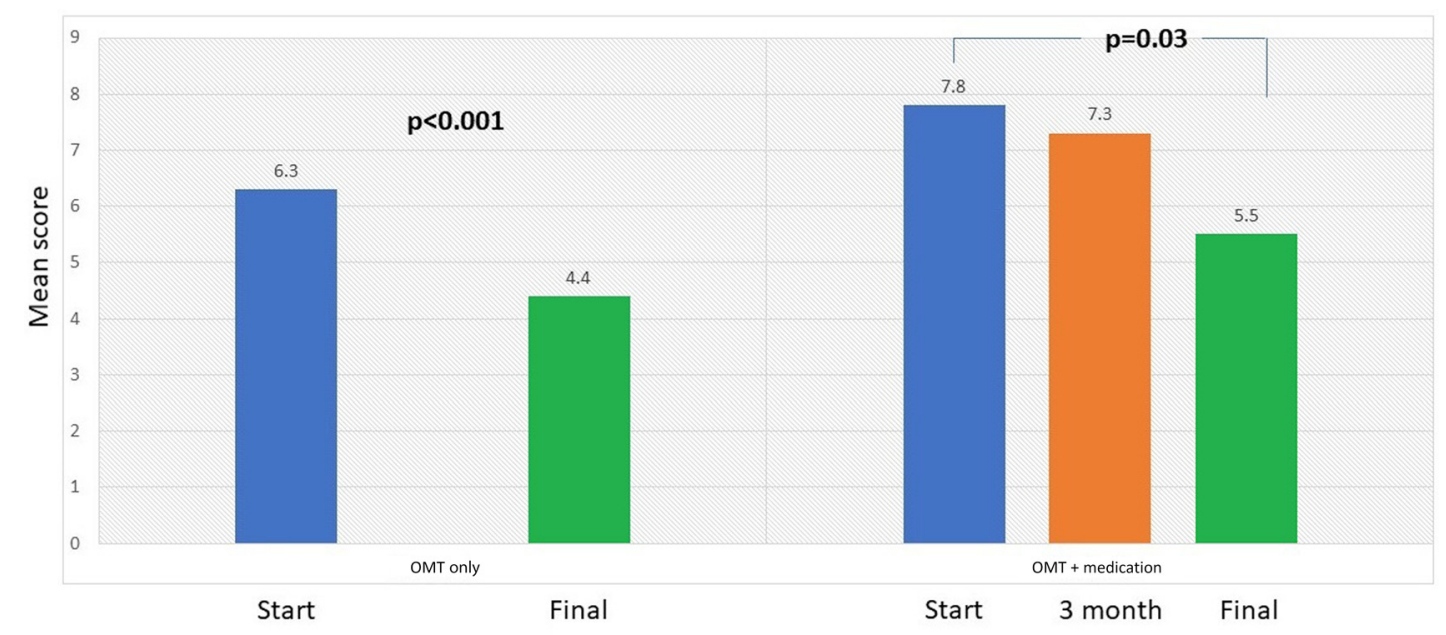

Figure 1: Mean Keele StarT scores between study groups (left, OMT only; right; OMT + medication). 


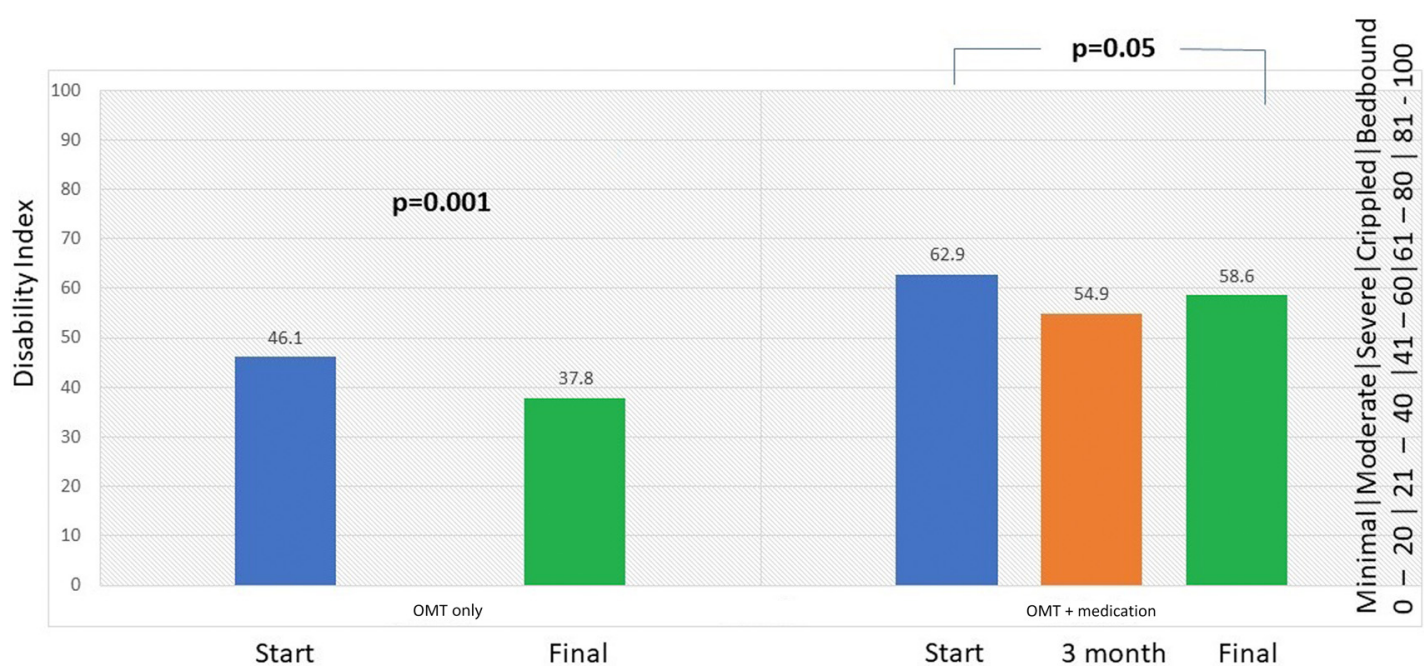

Figure 2: Mean Oswestry Disability Index scores between study groups (left, OMT only; right; OMT + medication).

of the study, at three months, and at the end of the study (one year).

\section{Results}

\section{Descriptive group comparisons}

Thirty-six patients were enrolled in the study. The groups did not differ on age $(t=0.7 ; \mathrm{p}=0.408)$ or gender $(\mathrm{x}=0.002$; $\mathrm{p}=0.647$ ). The patients' mean age was 46.7 years (SD, 13.7 years) and the study population included 25 women (69.4\%) and 11 men (30.6\%). At the start of treatment, the OMT-only group had significantly lower Oswestry Disability Index scores than the OMT + medication group $(t=9.034 ; p=0.005)$, while the OMT-only group had slightly lower Keele STarT scores than the OMT + medication group $(\mathrm{t}=2.8 ; \mathrm{p}=0.103)$ (Table 1).

\section{OMT-only group}

There were 26 patients in the OMT-only group with a mean age of 45.5 years (SD, 14.9 years). This group included 18 women (69.2\%) and eight men (30.8\%). The mean number of OMT visits for patients in this group was 5.3 (SD, 3.9). There was a 30\% relative decrease in the mean score for the Keele STarT survey within this group. These patients' mean score at enrollment was 6.3 (SD, 2.39) and 4.4 (SD, 2.30; $\mathrm{p}=<0.0001$ ) at the end of the study (Figure 1). The Oswestry Disability Index demonstrated an $18 \%$ relative decrease in disability index. These patients' mean disability score at enrollment was 46.1 (SD, 14.88) compared to 37.8 (SD, 11.6; $\mathrm{p}=0.001$ ) at the end of the study (Figure 2).

\section{OMT + medication group}

There were 10 patients in the OMT + medication group with a mean (SD) age of 49.8 (10.3) years. This group included seven women (70\%) and three men (30\%). The mean (SD) number of OMT visits for patients in this group was 4.3 (3.1). At the start of study, one patient used hydrocodone, five patients used cyclobenzaprine, and four patients used other medications. Within this group, a comparison was done from enrollment to three months into the study (prior to OMT). Results indicated no significant improvement in pain and function occurred during that time period. According at the Keele STarT survey data, the mean (SD) was 7.8 (2.39) at enrollment and 7.3 (3.0) after three months. However, at the end of one year, the mean (SD) was 5.5 (2.6), a relative decrease of $29.5 \%(\mathrm{p}=0.03)$ (Figure 1). Oswestry disability index demonstrated an $18 \%$ relative decrease in disability index. Overall, OMT + medication group went from a mean score of 62.9 (15.4) to 51.3 (18.0; $\mathrm{p}=0.05$ ) (Figure 2), although from enrollment to three months the decrease in the disability index was not significant (54.9-62.9; $\mathrm{p}=0.142$ ). The type of muscle relaxer was not restricted, but every patient who was prescribed a muscle relaxer was prescribed cyclobenzaprine. At the last visit, only two patients were using cyclobenzaprine and none were using hydrocodone. The number of patients in this group using cyclobenzaprine declined during the study from $5(50 \%)$ to $2(20 \%)$, a relative $60 \%$ decrease that was not statistically significant ( $\mathrm{p}=0.25)$.

Both groups improved similarly over time. There were no differences between groups on pain assessment over time (Keele STarT) $(\mathrm{p}=0.59)$ or on disability/function (Oswestry) $(\mathrm{p}=0.43)$ (Table 1). 


\section{Discussion}

This study was able to demonstrate a significant improvement in pain and function. Both groups improved over time and the improvements were similar for each. This is demonstrated by the similar relative improvements observed for each. There was a similar relative improvement for both groups on the Keele STarT of approximately 30\%. There was a similar relative improvement for both groups on the Oswestry of $18 \%$. This is consistent with the objective determination of somatic dysfunction by all treating physicians and subsequently the treatment they provided. Ease or range of movement, anatomic asymmetry, or positional change are impacted by OMT and would have been similarly applied in both groups.

All comparisons demonstrated statistical significance despite the small sample size ( $\mathrm{n}=10$; Table 1$)$, including the OMT-only group having a decrease in the disability index. This group went from severe disability score to moderate disability. The OMT + medication group went from crippled to severe disability. Additionally, it was observed that patients already taking pain medication, specifically cyclobenzaprine, were able to decrease the frequency of medication used in $20-50 \%$ of patients. This relatively large decrease, however, did not reach statistical significance which is likely due to the low sample size.

Previous studies have been limited in duration. Franke et al. $^{7}$ completed a systematic review of 307 studies from 1991 to 2014 of patients with LBP examining the evidence of benefits and harms of noninvasive treatments for LBP. None of the studies were longer than 90 days but are instructive. Overall, clinically significant effects of OMT were found for improving pain and function in acute and chronic LBP. In 10 of the studies with 12 comparison groups, six studies found significant effect with the use of OMT, three studies found a nonsignificant effect, and three studies found a nonsignificant effect in the control group. There was overall moderate quality evidence on improvement in overall pain and there was overall quality evidence in favor of OMT usage formoderate functional status.

The results of this study support current guidelines for the use of OMT as a first-line therapy for LBP without the use of pharmacotherapy. The initial design of this study was to determine whether OMT could improve pain and function in those with back pain and to also determine if a decrease in pain medications could be seen with the use of OMT. We assumed at the beginning of this study that more patients taking muscle relaxers and opioids would join this study. Consequently, acetaminophen and NSAIDs were not an exclusion criterion for the OMT-only group.
Unfortunately, there was not a large sample of patients using opioids within the study $(\mathrm{n}=1)$. This finding is likely because in recent years, our ambulatory clinic began limiting the use of opioid prescribing. The most frequently used pain medication in this study was muscle relaxers, specifically cyclobenzaprine, and therefore any conclusions regarding decreasing the use of opioids following OMT cannot be drawn from this study.

Limitations of our study included limited sample size, limited variety of pain medications, and multiple physician providers. There were 10 different physicians performing the OMT on patients. Some patients saw the same physician each time, while others saw a different physician. This physician differential may have diluted the strength of the effect that consistent monitoring and subsequent treatment frequency may have provided.

It is difficult to conduct a sham-controlled OMT study that extends over a one-year period for ethical reasons. According to Noll et al., "any form of touch may have an accompanying beneficial physiologic effect.” All sham interventions can be conceptualized as comparing one form of manipulation we consider effective with another form we consider ineffective. Therefore, sham-controlled trials may underestimate the actual benefit of manual therapy. It has been proposed that the ideal randomized clinical trial in manual medicine would include three arms: an active treatment group, a sham group, and a nontreatment group. However, such a study design is costly and requires a large number of participants for adequate statistical power. This was not possible in the clinical setting in which this trial was completed, but is a possibility for future research.

Looking forward, future studies may consider ensuring that patients see one physician for the entirety of their treatment. There could also be specific treatments for a specific somatic dysfunction, so all patients receive the same type of OMT based on their somatic dysfunction. A control group in which patients were only using one type of a pain medication could be considered (e.g., future studies could examine opioids and OMT alone). Patients already taking opioids could be started on OMT and compared with a group of patients on opioids but not receiving OMT (control group). Patients would be followed for pain and function improvements and for decreases in opioid use and dosage.

\section{Conclusion}

The current study demonstrated that both OMT-only and OMT + medication groups showed improvement in pain 
and disability. OMT can be used as a tool to help alleviate pain and improve function in patients with chronic LBP who are not currently using prescribed pain medications for back pain. OMT can also be used to titrate down pain medication for patients while still providing treatment that will help alleviate pain.

Research funding: None reported.

Author contributions: All authors provided substantial contributions to conception and design, acquisition of data, or analysis and interpretation of data; all authors drafted the article or revised it critically for important intellectual content; all authors gave final approval of the version of the article to be published; and all authors agree to be accountable for all aspects of the work in ensuring that questions related to the accuracy or integrity of any part of the work are appropriately investigated and resolved.

Competing interests: Authors state no conflict of interest. Informed consent: Informed consent was obtained from all individuals included in this study.

Ethical approval: Research involving human subjects complied with all relevant national regulations, institutional policies and is in accordance with the tenets of the Helsinki Declaration (as revised in 2013), and has been approved by the authors' Institutional Review Board (Ascension Genesys) or equivalent committee.

\section{References}

1. Koes BW, van Tulder MW, Thomas S. Diagnosis and treatment of low back pain. BMJ. 2006;332(7555):1430-1434. doi: 10.1136/bmj. 332.7555.1430.

2. Finley CR, Chan DS, Garrison S, et al. What are the most common conditions in primary care? Systematic review. Can Fam Physician. 2018;64(11):832-840.

3. Snow RJ, Seffinger MA, Hensel KL, Wiseman R. American Osteopathic Association Guidelines for Osteopathic Manipulative Treatment for Patients with Low Back Pain: Taskforce on the Low Back Pain Clinical Practice Guidelines. J Am Osteopath Assoc. 2016;116:536-54. doi: 10.7556/jaoa.2016.107.

4. National Institute on Drug Abuse. Opioid Overdose Crisis. https://www.drugabuse.gov/drugs-abuse/opioids/opioidoverdose-crisis. Updated January 2019. Accessed June 12, 2019.

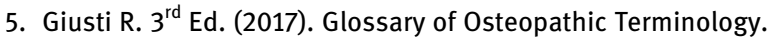
Educational Council on Osteopathic Principles (ECOP) of the Am Assoc of Colleges of Osteopathic Medicine (AACOM). Chevy Chase, MD: AACOM.
6. Sposato NS, Bjerså K. Osteopathic manipulative treatment in surgical care: short review of research publications in osteopathic journals during the period 1990 to 2017. J Evid Based Integr Med. 2018;23:25. doi: 10.1177/2515690X18767671.

7. Franke H, Franke J, Fryer G. Osteopathic manipulative treatment for nonspecific low back pain: a systematic review and metaanalysis. BMC Musculoskelet Disord. 2014;15:286. doi:10.1186/ 1471-2474-15-286.

8. Rubinstein SM, Terwee CB, Assendelft WJJ, et al. Spinal manipulative therapy for acute lowback pain. Cochrane Database Syst Rev. 2012;9:CD008880. doi: 10.1002/14651858.CD008880. pub2.

9. Licciardone JC, Stoll ST, Fulda KG, et al. Osteopathic manipulative treatment for chronic low back pain: a randomized controlled trial. Spine. 2003;28(13):1655-62. doi: 10.1186/ 1471-2474-6-43.

10. Cruser dA, Maurer D, Hensel K, Brown S, White K, Stoll S. A randomized, controlled trial of osteopathic manipulative treatment for acute low back pain in active duty military personnel. J Man Manip Ther. 2012;20:5-15. doi: 10.1179/ $2042618611 Y .0000000016$.

11. Arguisuelas MD, Lisón JF, Sánchez-Zuriaga D, Martínez-Hurtado I, Doménech-Fernández J. Effects of myofascial release in nonspecific chronic low back pain: a randomized clinical trial. Spine. 2017; 42(9):627-634. doi: 10.1097/BRS.0000000000001897.

12. Noll DR, Degenhardt BF, Stuart M, et al. Effectiveness of a sham protocol and adverse effects in a clinical trial of osteopathic manipulative treatment in nursing home patients. J Am Osteopath Assoc. 2004;104(3):107-113.

13. Andersson GBJ, Lucente T, Davis AM, Kappler RE, Lipton JA, Leurgans S. A comparison of osteopathic spinal manipulation with standard care for patients with low back pain. $N$ Eng J Med. 1999;341:1426-1431. doi: 10.1056/NEJM199911043411903.

14. Heinze G: Unpublished D.O. Thesis. The Effectiveness of a Holistic Osteopathic Treatment in Subacute Low Back Pain. A Randomized Controlled Trial. 2006, Akademie für Osteopathie, http://www. osteopathic-research.com/index.php?option=com_ jresearch\&view=publication\&task=show\&id=13797\&lang=en.

15. Licciardone J, Minotti DE, Gatchel RJ, Kearns CM, Singh KP. Osteopathic manual treatment and ultrasound therapy for chronic low back pain: a randomized controlled trial. Ann Fam Med. 2013;11(2):122-129. doi: 10.1370/afm.1468.

16. Licciardone JC, Kearns CM, Minotti DE. Outcomes of osteopathic manual treatment for chronic low back pain according to baseline pain severity: results from the OSTEOPATHIC Trial. Manual Therapy. 2013;18(6):533-540. doi: 10.1016/j.math.2013.05.006.

17. DiGiovanna EL. Introduction to Osteopathic Medicine. DiGiovanna EL, Schiowitz S, Dowling DJ, eds. An Osteopathic Approach to Diagnosis and Treatment. $3^{\text {rd }}$ ed. Philadelphia, PA: Lippincott Williams \& Wilkins; 2005:1.

18. Treager A, McAuley JH. STarT Back Screening Tool. J Physiother. 2013;59(2):131. doi: 10.1016/S1836-9553(13)70170-X.

19. Fairbank JC, Couper J, Davies JB, et al. The Oswestry Low Back Pain Disability Questionnaire. Physiotherapy. 1980;66(8): 271-73. 